\title{
Dysregulated miR-27a-3p promotes nasopharyngeal carcinoma cell proliferation and migration by targeting Mapk10
}

\author{
LIHUA LI $^{1}$ and ZHAOHUI LUO ${ }^{2}$ \\ ${ }^{1}$ Department of Radiotherapy, Hunan Cancer Hospital, Changsha, Hunan 410013; \\ ${ }^{2}$ Department of Neurology, Xiangya Hospital, Central South University, Changsha, Hunan 410008, P.R. China
}

Received November 6, 2016; Accepted January 16, 2017

DOI: 10.3892/or.2017.5544

\begin{abstract}
RNA-27a-3p is an important regulator of carcinogenesis and other pathological processes. However, its role in laryngeal carcinoma is still unknown. In our previous research, we found that miR-27a-3p expression was upregulated in nasopharyngeal carcinoma (NPC) using a microarray chip. In the present study, we identified miR-27a-3p as an endogenous promoter of metastatic invasion. The expression levels of miR-27a-3p were correlated with human metastatic progression outcomes and Kaplan-Meier survival. In silico database analyses revealed that Mapk10 is a potential target of miR-27a-3p, and luciferase reporter assay results revealed that miR-27a-3p directly inhibits the Mapk10 3' untranslated region (3'UTR). Real-time PCR and western blotting results ascertained that Mapk10 expression was regulated by miR-27a-3p. In addition, miR-27a-3p gain-of-function promoted cell proliferation, migration and invasion in 5-8 F NPC cells. These effects partially depended on Mapk10, and loss of miR-27a-3p function had the opposite effects.
\end{abstract}

\section{Introduction}

Aberrant expression of microRNAs (miRNAs) and of factors in their biogenesis have been frequently observed in different types of cancer (1). Disruption of miRNA expression patterns is now recognized as a hallmark of human cancer (2-4). Each miRNA is estimated to regulate an average of several hundred protein-coding genes, and $\sim 60 \%$ of proteins in cells are thought to be regulated by miRNAs $(5,6)$. Changes in miRNA expression patterns have been linked to profound effects on cell phenotypes, and miRNAs have an emerging role in diverse physiological and pathological processes (7). Notably, the dysregulation of miR-27a in various types of cancer suggests

Correspondence to: Dr Zhaohui Luo, Department of Neurology, Xiangya Hospital, Central South University, 87 Xiangya Road, Kaifu, Changsha, Hunan 410008, P.R. China

E-mail: luozhaohui_xy@126.com

Key words: miR-27a-3p, Mapk10, nasopharyngeal carcinoma, metastatic invasion, proliferation it plays an important role in the occurrence and progression of cancer. Zhou et al revealed that miR-27a-3p functions as an oncogene in gastric cancer by targeting BTG2 (8). miR-27 may also play a key role in osteoblast proliferation, apoptosis and differentiation through the post-transcriptional regulation of sFRP1 (9). Additionally, miR-27a/b promoted angiogenesis by targeting the angiogenesis inhibitor SEMA6A, which controls the repulsion of neighboring endothelial cells (10).

Research on nasopharyngeal carcinoma (NPC) has mirrored other cancer research programs. Studies have largely focused on molecular defects, including the dysregulation of miRNAs. To date, several miRNAs have been shown to target specific mRNAs to regulate the progression of NPC. miR-216b (11), miR-18a (12), miR-218 (13), miR-26a/b (14,15), miR-10b (16), let-7 (17), miR-141 (18) and miR-200a (19) have been shown to possess tumor-suppressive functions in NPC. Not surprisingly, Epstein-Barr virus-encoded miRNAs have oncogenic properties (20-22). Our previous research described the roles of miR-18a in the progression of NPC and the mechanism by which miR-18a promotes malignant tumor progression. Effort has also been made to associate miR-18a and Dicer with NPC (12). miR-27a-3p was revealed to be upregulated in miRNA expression profile studies from two independent laboratories. Using deep sequencing screen laser-microdissected biopsies from 12 NPC and 8 chronic nasopharyngitis patients, Zhang et al identified miR-27a-5p as differentially expressed (23). In a previous study, we also demonstrated that miR-27a-3p was significantly upregulated during NPC progression (24), suggesting an oncogenic role for miR-27a-3p in NPC.

TargetScan, PicTar, miRNAome and miRanda predicted thousands of target genes for miR-27a-3p. The method used to select putative target genes of miR-27a-3p is another highlight of the present study. Since miRNAs downregulate gene expression by targeting the 3' untranslated region (3'UTR) of mRNAs $(24,25)$, NPC cDNA expression data (GSE12452) were downloaded from the publicly accessible National Center for Biotechnology Information-Gene Expression Omnibus (GEO). We identified Mapk10 as a target gene of miR-27a-3p, and the cDNA data revealed that Mapk10 expression was downregulated in NPC.

In the present study, we demonstrated that miR-27a-3p, which was predicted to target Mapk10 in silico, was upregulated in tumors of various origins and in the 5-8 F cell line. 
Furthermore, we determined that Mapk10 expression was inversely correlated with miR-27a-3p expression, suggesting that miR-27a-3p may regulate Mapk10. Our results indicated that miR-27a-3p directly binds to and downregulates Mapk10 levels in vitro. Importantly, the miR-27a-3p-mediated increase in NPC cell proliferation, migration and invasion partially depended on Mapk10. Altogether, our results demonstrated that $\mathrm{miR}-27 \mathrm{a}-3 \mathrm{p}$ regulates Mapk10 and provide evidence for the development of novel cancer therapies.

\section{Materials and methods}

Cell lines and human subjects. The NPC cell lines 5-8 F, 6-10 B and HK-1 (Cancer Research Institute of Central South University, Changsha, China) were cultured in RPMI1640 medium supplemented with penicillin G $(100 \mathrm{U} / \mathrm{ml})$, streptomycin $(100 \mathrm{mg} / \mathrm{ml})$ and $10 \%$ fetal calf serum (FCS). Immortalized normal nasopharynx epithelial NP69 cells were cultured in RPMI-1640 medium supplemented with penicillin G (100 U/ml), streptomycin $(100 \mathrm{mg} / \mathrm{ml}), 10 \%$ FCS and growth factors. The cells were grown at $37^{\circ} \mathrm{C}$ in a humidified atmosphere of $5 \% \mathrm{CO}_{2}$ and were routinely sub-cultured using $0.25 \%(\mathrm{w} / \mathrm{v})$ trypsin-EDTA solution.

Ethical standards. All procedures were in accordance with the standards of the relevant Ethics Committees for human experimentation (institutional and national) and with the 1964 and later versions of the Declaration of Helsinki.

Vectors, oligonucleotides, antibodies and siRNA. Synthetic miRNA mimics and 2'-O-methyl-modified inhibitors, 'antagomiRs', were purchased from the GenePharma Company (Shanghai, China). An miR-insensitive Mapk10 construct was purchased from GeneCopoeia Company (Guangzhou, China). The rabbit anti-human Mapk10 antibody was purchased from Cell Signaling Technology, Inc. (Beverly, MA, USA). The Mapk10 siRNA (S11163) was purchased from Life Technologies Company (Shanghai, China). The Mapk10-3 siRNA produced the best results and was used in subsequent experiments.

Quantitative real-time PCR. Expression of the miRNAs was evaluated using SYBR-Green quantitative real-time RT-PCR (Takara, Dalian, China). Total RNA was extracted from cells and samples using TRIzol ${ }^{\circledR}$ reagent (Invitrogen, Carlsbad, CA, USA). The individual miRNA qPCR Quantitation kits were purchased from GenePharma Company. The quantitative PCR was performed according to the manufacturer's instructions. The qPCR cycle was $98^{\circ} \mathrm{C}$ for $2 \mathrm{~min}, 40$ cycles of $95^{\circ} \mathrm{C}$ for $15 \mathrm{sec}$, and $60^{\circ} \mathrm{C}$ for $30 \mathrm{sec}$. A final melting curve analysis $\left(60-95^{\circ} \mathrm{C}\right)$ was included. The standard curve had a slope of $\sim-3.32$ ( $\sim 100 \%$ efficiency). The miRNA PCR results were quantified using the $2^{-\Delta \Delta \mathrm{Ct}}$ method against U6 for normalization. The mRNA PCR quantification used the $2^{-\Delta \Delta \mathrm{Ct}}$ method against GAPDH for normalization. The data are representative of the means of 3 experiments. The following RT-PCR primers were used: miR-27a-3p F, TTCACAGTGGCTAAGTTCCGC; Mapk10 F, CTTCCCAGATTCCCTCTTCC and Mapk10 R, GCTGGGTCATACCAGACGTT; GAPDH F, GAGTCAAC GGATTTGGTCGT and GAPDH R, TTGATTTTGGAGG GATCTCG.
MTT assay. Cells (5-8 F) were seeded at a density of $2 \times 10^{3}$ cells/well in 96 -well plates $24 \mathrm{~h}$ before transfection. The cells were incubated in growth medium for $24 \mathrm{~h}$. A volume of $20 \mu \mathrm{l}$ of MTT solution $(5 \mathrm{mg} / \mathrm{ml})$ was added to each well, and the plates were incubated at $37^{\circ} \mathrm{C}$ for an additional $4 \mathrm{~h}$. The media was removed, and $150 \mu \mathrm{l}$ dimethyl sulfoxide (DMSO) was added to each well. The plates were shaken for $10 \mathrm{~min}$ to dissolve the MTT formazan crystals. The optical density (OD) of each well was determined with a scanning multi-well spectrophotometer at a wavelength of $490 \mathrm{~nm}$. The experiment was repeated 3 times, and 6 parallel samples were assessed each time.

Wound closure assay. The cells were transfected with synthetic miRNA mimics or the 2'- $O$-methyl-modified inhibitors in a 6 -well dish until they reached $90 \%$ confluency. A wound was created using a sterile $10-\mu$ l pipette tip, and then the dish was washed with $1 \mathrm{X}$ phosphate-buffered saline (PBS) to remove detached cells. Subsequently, the cells were cultured in medium with $2 \%$ serum and treated with $5 \mathrm{mM}$ hydroxyurea (26). Migration at the wound site was documented using a microscope (Nikon Corporation, Tokyo, Japan) at different time-points (0, 24 and $48 \mathrm{~h})$.

Transwell migration assay. Before the cells were seeded, Corning Costar Transwell 24-well plates (8- $\mu \mathrm{m}$ pores) Corning, Corning, NY, USA) were coated with Matrigel (BD Biosciences, Franklin Lakes, NJ, USA) and placed in the cell culture hood for $1 \mathrm{~h}$ at $37^{\circ} \mathrm{C}$. A total of $1 \times 10^{5}$ cells were seeded in the wells after they were transfected, and were cultured in medium with $2 \%$ serum. Normal growth medium was placed in the bottom wells. The cells were then allowed to migrate for 24 or $48 \mathrm{~h}$. The migrated cells were fixed with $100 \%$ methanol for $1 \mathrm{~min}$ and allowed to air dry. The cells that invaded the lower surface of the membrane were stained by dipping the inserts in a staining solution for $20 \mathrm{~min}$. The stained cells were then counted.

Western blotting assay. The protein used for western blotting was extracted using mRIPA buffer containing protease inhibitors $(50 \mathrm{mM}$ Tris, $\mathrm{pH} 7.4 ; 100 \mathrm{mM} \mathrm{NaCl} ; 1 \%$ Nonidet $\mathrm{P}-40 ; 0.5 \%$ deoxycholic acid; $0.1 \% \mathrm{SDS} ; 10 \mu \mathrm{g} / \mathrm{ml}$ aprotinin; $10 \mu \mathrm{g} / \mathrm{ml}$ leupeptin; and $1 \mathrm{mM}$ PMSF). The proteins were quantified using the $\mathrm{BCA}^{\mathrm{TM}}$ protein assay kit (Pierce, Rockford, IL, USA). The western blotting system was established using a Bio-Rad Bis-Tris gel system according to the manufacturer's instructions (Bio-Rad, Hercules, CA, USA). The primary antibodies were prepared in 5\% blocking buffer. The primary antibody (against Mapk10 or GAPDH) was incubated with the membrane overnight at $4^{\circ} \mathrm{C}$. The membrane was then briefly washed, and incubated with a secondary antibody for $1 \mathrm{~h}$ at room temperature. Finally, a 40:1 peroxide:luminol solution was added to cover the blot surface, and the blot was incubated for $5 \mathrm{~min}$ at room temperature. The signals were captured and the band intensity was quantified using the Bio-Rad ChemiDoc XRS system.

Luciferase assay. Human Mapk10 oligonucleotides (44-60 bp), in which miR-27a-3p binding sites were present (WT) or deleted (MUT), were inserted into the SpeI and HindIII sites of the multiple cloning sites in the pMIR-REPORT vector to create recombinant plasmids according to the manufacturer's 


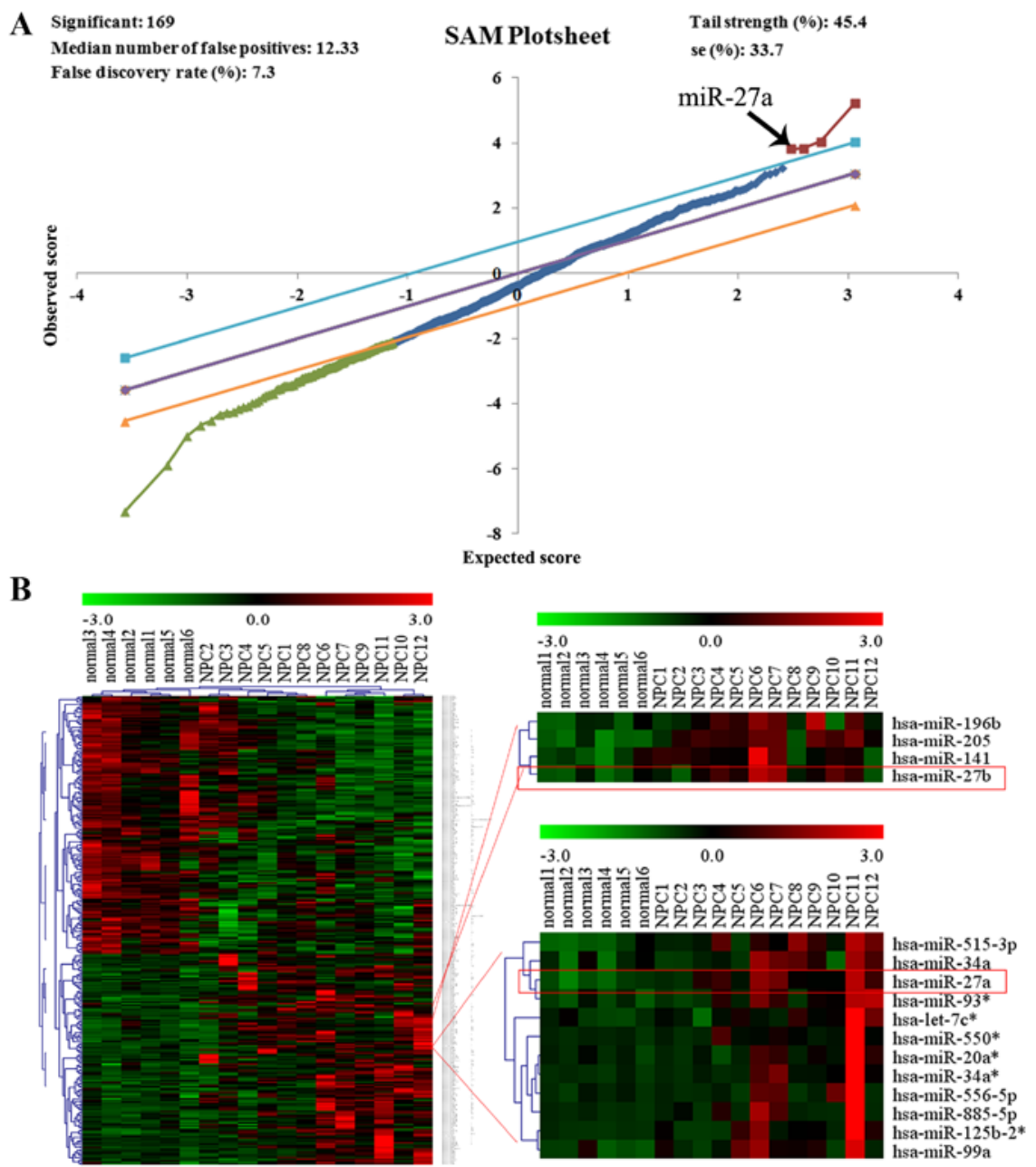

Figure 1. miRNA microarray analysis of 12 NPC cases and 6 control samples of healthy nasopharyngeal epithelial tissue. (A) SAM was determined using significance analysis of microarray data. (B) miRNA data were clustered by Cluster3.0. Red represents high expression, and green represents low expression NPC, nasopharyngeal carcinoma.

instructions. The oligonucleotide sequences used were as follows: WT Mapk10 F, CTAGTAAAGTGCTTTCCTGAGC ACTTAAGGAGGCACTGTGAAACATCTTGGTTATGTA CAAGA; WT Mapk10 R, GCTTTCTTGTACATAACCAAG ATGTTTCACAGTGCCTCCTTAAGTGCTCAGGAAAGC ACTTTA; MUT Mapk10 F, CTAGTAAAGTGCTTTCCTG AGCA-GGAGGC-ACATCTTGGTTATGTACAAGA; and MUT Mapk10 R, GCTTTCTTGTACATAACCAAGATGT-G CCTCCT-GCTCAGGAAAGCACTTTA.

Cells $(5-8 \mathrm{~F})$ were seeded at $5 \times 10^{4}$ cells/well in RPMI-1640 medium in a $24-w e l l$ plate. miR-27a-3p or miR-nc (50 nM), along with $0.4 \mu \mathrm{g}$ pMIR-REPORT luciferase plasmid containing WT-Mapk10 or MUT-Mapk10 oligonucleotides (as aforementioned) and $0.08 \mu \mathrm{g}$ pRL-TK plasmid, were cotransfected into 5-8 $\mathrm{F}$ cells using Attractene transfection reagent (Qiagen, Hilden, Germany). After a 48-h incubation, the cells were lysed in cell culture luciferase lysis buffer (CLLB), and the luciferase activity was analyzed using a Dual-Luciferase Reporter Assay System (Promega, Madison, WI, USA). The ratio of Renilla luciferase to firefly luciferase was calculated. All experiments were performed in triplicate.

Clinical specimens. Snap-frozen NPC biopsies were obtained from NPC patients. Healthy nasopharyngeal epithelial samples from biopsy-negative cases were used as a control. The samples were obtained from Hunan Cancer Hospital, Central South University. The patients were informed concerning sample collection and signed informed consent forms. Collection and use of the tissue samples was approved by the Ethical Review Committees of Hunan Cancer Hospital.

Statistical analysis. Survival data were analyzed using KaplanMeier analyses. The log-rank and $\chi^{2}$ tests were used to identify differences between survival curves according to miR-27a-3p and Mapk10 status. The data, including those from the qRT-PCR experiments, are represented as the mean \pm standard deviation. The Mann-Whitney U test was used to compare the expression of miR-27a-3p in different NPC stages. The intensity values from the miRNA array were analyzed by SAM. The statistical analyses were performed using SPSS 22.0. All p-values were two-sided, and p-values $<0.05$ were considered significant. Cronbach's $\alpha$ was calculated using SPSS and used to evaluate agreement between the two pathologists.

\section{Results}

Expression of miR-27a-3p in the NPC samples and cell lines. In our previous study, we investigated miRNA expression in NPC 

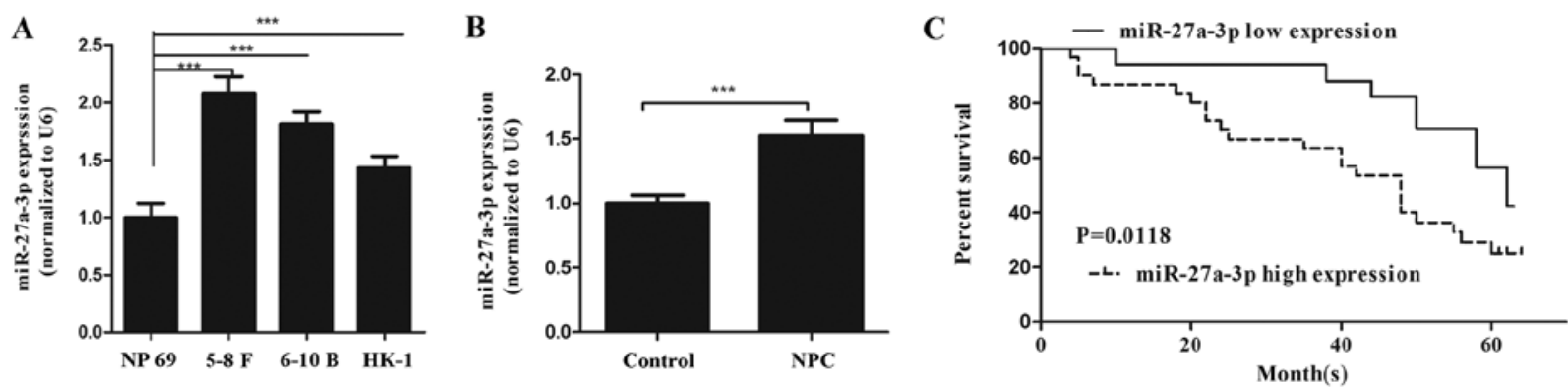

Figure 2. Expression level of miR-27a-3p in NPC samples and cell lines. (A) RT-PCR analysis of miR-27a-3p was performed in the 5-8 F, 6-10B and HK-1 cell lines and in the immortalized nasopharyngeal epithelial cell line NP69 $\left({ }^{* * *} \mathrm{p}<0.001\right)$. Compared to that in the control, the expression of miR-27a-3p was clearly upregulated in the 5-8 F, 6-10B and HK-1 cell lines. (B) Real-time RT-PCR analysis of miR-27a-3p was performed for 68 NPC patient samples and 21 control samples $\left({ }^{* * *} \mathrm{p}<0.001\right)$. Compared to that in the control samples, the expression of miR-27a-3p was clearly upregulated in the NPC samples. The expression of miR-27a-3p was normalized to U6. The data are presented as the mean \pm SD of 3 replicates. (C) Kaplan-Meier curves are shown for patients according to tumor expression of miR-27a-3p. Differences among the survival curves are shown. NPC death was significantly associated with the expression of miR-27a-3p. Lower overall patient survival $(n=48)$ was associated with higher miR-27a-3p expression $(\mathrm{p}<0.05)$. NPC, nasopharyngeal carcinoma.

samples using microarray chips (24). In the present study, we reanalyzed the miRNAs array data by SMA (Fig. 1A) and then clustered the miRNA data using Cluster3.0, as shown in Fig. 1B. Using dendrogram-based methods for clustering, compared to 6 samples of healthy nasopharyngeal epithelial tissue, miR27a-3p was upregulated in 12 NPC samples (Fig. 1B). In the present study, we evaluated miR-27a-3p expression in 68 NPC and 21 healthy nasopharyngeal epithelial tissue samples by quantitative real-time PCR. We also confirmed that miR$27 a-3 p$ was associated with the progression of NPC, as the expression of miR-27a-3p in NPC samples was increased when compared to the healthy control samples (Fig. 2B) and in addition gradually increased with clinical stage (Table I). We also examined the expression of miR-27a-3p in the 5-8 F, 6-10 B and HK-1 NPC cell lines (Fig. 2A). This analysis ascertained that the expression of miR-27a-3p was greater in the NPC cell lines compared to the healthy nasopharynx epithelial cell line NP69. Kaplan-Meier plots were constructed using the real-time PCR results $(n=48)$. Increased survival was associated with low miR-27a-3p expression [Fig. 2C; hazard ratio, $0.4289 ; 95 \%$ confidence interval (CI), 0.8465-1.737; $\mathrm{p}=0.0118$ ], indicating that death from NPC was associated with high levels of miR-27a-3p $(\mathrm{p}<0.05)$. Subsequently, we analyzed the association of the characteristics of 68 NPC samples, including age, gender, lymph node metastasis, clinical stage and EBV (VCA-IgA), with miR-27a-3p expression (Table I). This analysis indicated that miR-27a-3p expression was not associated with age or gender but was associated with lymph node metastasis, clinical stage and EBV (VCA-IgA). From these data, we determined that miR-27a-3p expression was upregulated in NPC samples and cell lines and was correlated with NPC survival, lymph node metastasis, clinical stage and EBV (VCA-IgA). These results indicate that this molecule is involved in the progression of NPC.

miR-27a-3p promotes $5-8 \mathrm{~F}$ cell proliferation, migration and invasion in vitro. To explore the role of miR-27a-3p in NPC 5-8 F cells, we transfected miR-27a-3p mimics (miR-27a-3p) and negative control (miR-nc) or miR-27a-3p inhibitors (anti-miR-27a-3p) and inhibitor control (anti-nc) into 5-8 F cells. MTT, cell wound closure and Transwell with Matrigel (BD Biosciences) assays were performed in vitro to determine
Table I. The clinical data of miR-27a-3p expression in NPC patients $(n=68)$.

\begin{tabular}{|c|c|c|c|c|c|}
\hline \multirow{2}{*}{$\begin{array}{l}\text { Clinicopatho- } \\
\text { logical } \\
\text { features }\end{array}$} & \multirow[b]{2}{*}{$\mathrm{n}$} & \multicolumn{2}{|c|}{ miR-27-3p } & \multirow[b]{2}{*}{$\chi^{2}$} & \multirow[b]{2}{*}{ P-value } \\
\hline & & $\begin{array}{l}\text { High } \\
(3-4)\end{array}$ & $\begin{array}{l}\text { Low } \\
(0-2)\end{array}$ & & \\
\hline Sex & & & & 1.434 & 0.231 \\
\hline Male & 38 & 28 & 10 & & \\
\hline Female & 30 & 18 & 12 & & \\
\hline Age (years) & & & & 0.249 & 0.618 \\
\hline$\geq 50$ & 42 & 20 & 22 & & \\
\hline$<50$ & 26 & 14 & 12 & & \\
\hline $\begin{array}{l}\text { Lymph node } \\
\text { metastasis }\end{array}$ & & & & 7.857 & 0.005 \\
\hline Yes & 46 & 35 & 9 & & \\
\hline No & 22 & 10 & 12 & & \\
\hline Clinical stage & & & & 11.118 & $0.01^{\mathrm{a}}$ \\
\hline I-II & 25 & 7 & 18 & & \\
\hline III-IV & 43 & 30 & 13 & & \\
\hline EBV (VCA-IgA) & & & & 5.427 & 0.02 \\
\hline$\geq 1: 10^{+}$ & 53 & 46 & 7 & & \\
\hline$\leq 1: 10^{+}$ & 15 & 9 & 6 & & \\
\hline
\end{tabular}

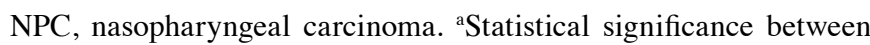
clinical stage I-II and III-IV.

the roles of miR-27a-3p in the development of NPC, including its effect on cell growth, migration and invasion. The MTT assay revealed that miR-27a-3p overexpression increased cell viability. In contrast, the miR-27a-3p inhibitor (antimiR-27a-3p) obviously inhibited growth compared to the inhibitor control (Fig. 3A). The wound healing (Fig. 3B) and Transwell invasion assays (Fig. 3C and D) showed the same result; compared to the controls, miR-27a-3p promoted NPC cell proliferation, migration and invasion in vitro. In contrast, the miR-27a-3p inhibitor obviously inhibited the growth and invasion of NPC cells. 
A

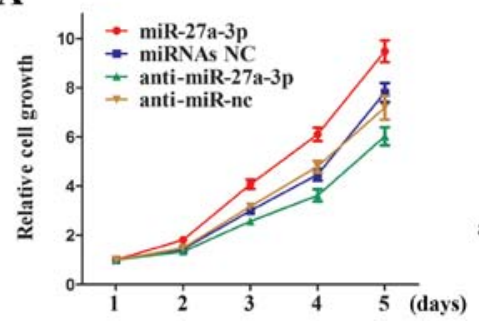

C

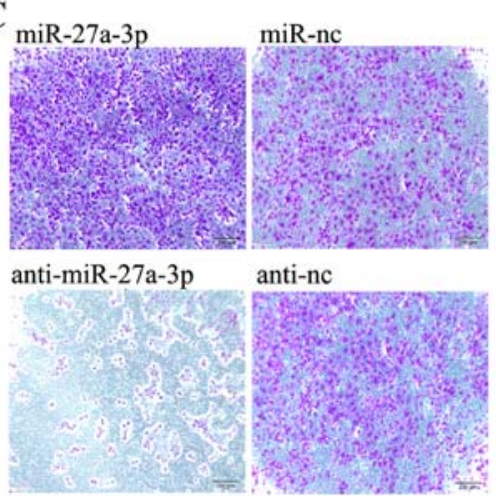

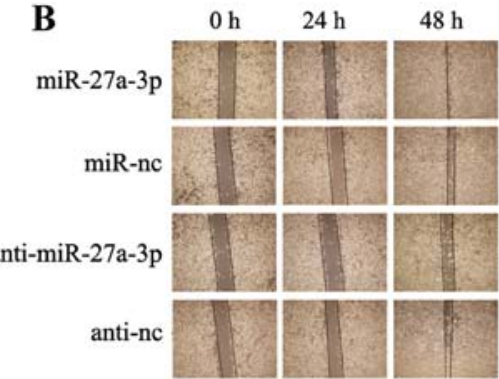

D

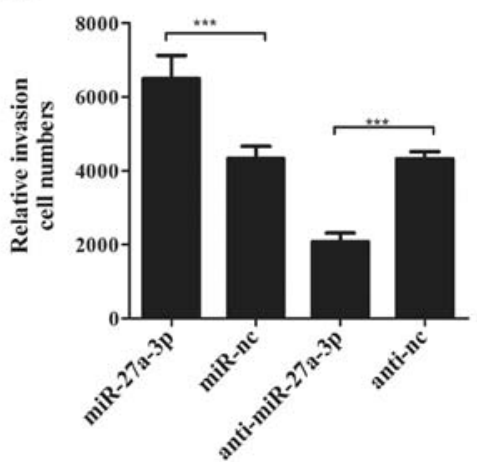

Figure 3. miR-27a-3p promotes tumor cell growth and invasion in vitro. (A) miR-27a-3p promoted tumor cell growth. The MTT cell viability assay revealed that miR-27a-3p increased cell viability. The MTT assay was performed after transfection of miR-27a-3p mimics or an miR-27a-3p inhibitor into the 5-8 F cells. Overexpression of miR-27a-3p increased 5-8 F cell proliferation. The miR-27a-3p inhibitor significantly inhibited the growth of 5-8 F cells. The data represent the mean values of 3 experiments, each performed in triplicate. The data are shown as the mean $\pm \mathrm{SD}$. (B) miR-27a-3p promoted tumor cell migration in vitro. The wound healing assay revealed that miR-27a-3p promoted the migration and mobility of 5-8 F cells. Overexpression of miR-27a-3p using mimics clearly promoted wound gap closure in a time-dependent manner. Knockdown of miR-27a-3p using chemically synthesized miR-27a-3p inhibitors delayed wound gap closure. (C) The Transwell migration assay revealed that miR-27a-3p increased invasion ability. Representative images of the migrated stained cells are shown. (D) Cells were counted in five randomly selected areas, and statistical analyses were performed using SPSS 22.0. The data are shown as the mean $\pm \mathrm{SD}\left({ }^{* * * *} \mathrm{p}<0.001\right.$; two-sided Student's t-test). The experiment was repeated in 5-8 F cells.

A

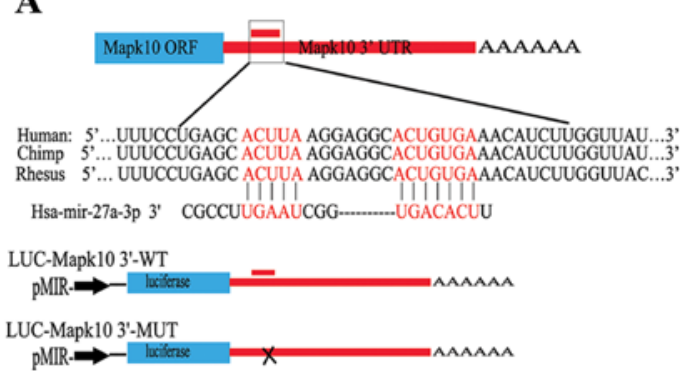

B

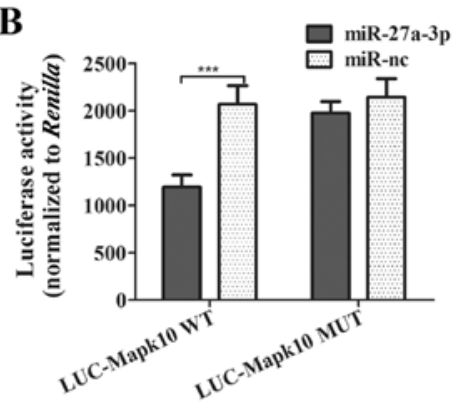

C

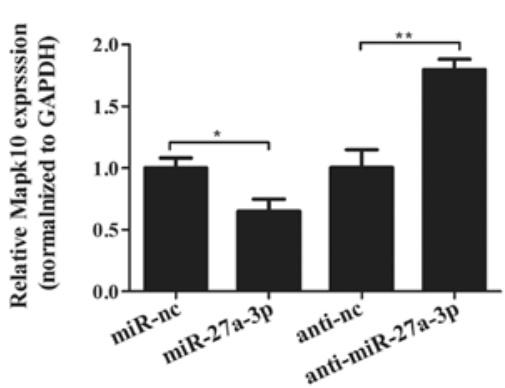

D

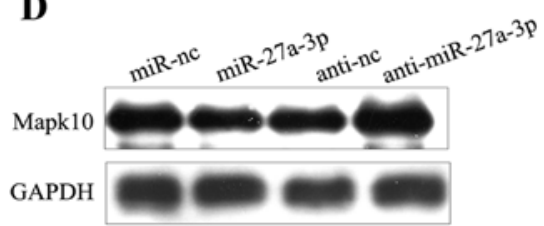

Figure 4. miR-27a-3p targets Mapk10 and downregulates endogenous Mapk10 expression in 5-8 F cells. (A) Schematic representation of the 3'UTR of Mapk10. The red bars show the predicted miR-27a-3p binding sites in the 3'UTR of Mapk10. The mature miR-27a-3p sequence is shown aligned to the target sites. LUC-Mapk10-WT represents the Mapk10 3'UTR containing miR-27a-3p binding sites (WT); LUC-Mapk10-MUT represents the Mapk10 3'UTR in which the miR-27a-3p binding sites were deleted (MUT). (B) Luciferase activity assay. The reporter constructs, in which the Mapk10 3'UTR was either the wild-type form or was missing the miR-27a-3p binding sites (LUC-Mapk10-WT or LUC-Mapk10-MUT), were cloned downstream of the luciferase open reading frame. Cells $(5-8 \mathrm{~F})$ were co-transfected with a luciferase construct and miR-27a-3p mimics or control miRNA. Renilla constructs were also cotransfected as an internal control. Luciferase activity was normalized to Renilla luciferase activity. The data are presented as the mean \pm SD of two experiments with 6 replicates (Student's t-test, ${ }^{* * *} \mathrm{p}<0.001$ ). (C) miR-27a-3p downregulated the endogenous expression of Mapk10, as shown by real-time PCR. Real-time PCR for Mapk10 was performed $48 \mathrm{~h}$ after the transfection of miR-27a-3p mimics or their inhibitor. GAPDH was used as an internal control (Student's t-test; " $\mathrm{p}<0.05$, "** $\mathrm{p}<0.01$ ). (D) Endogenous Mapk10 protein expression was downregulated by miR-27a-3p. The results were similar to those obtained with real-time PCR. 
A

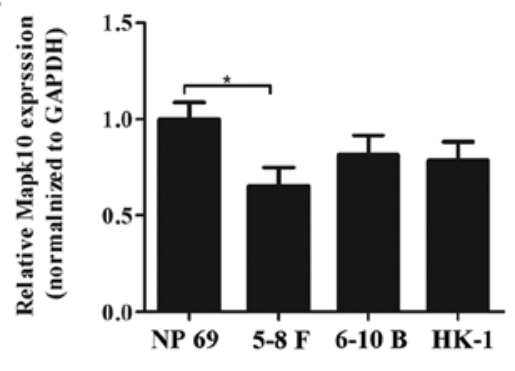

D

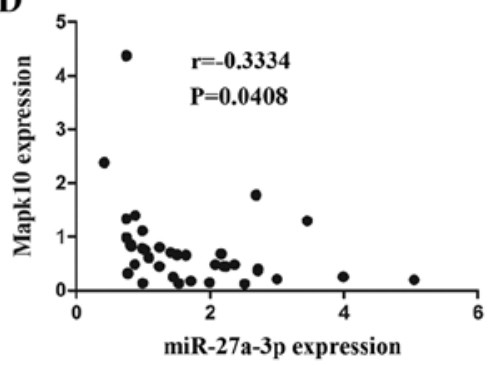

B

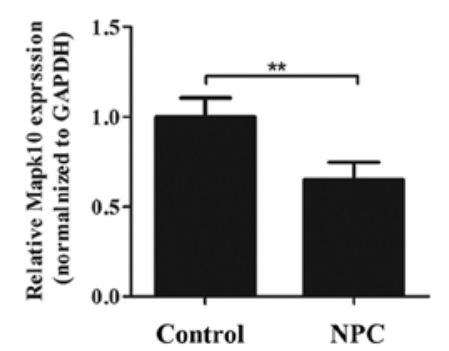

C

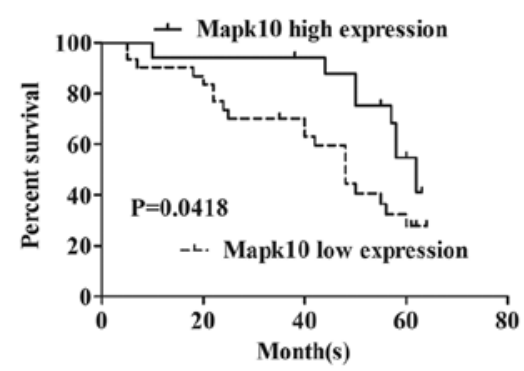

E

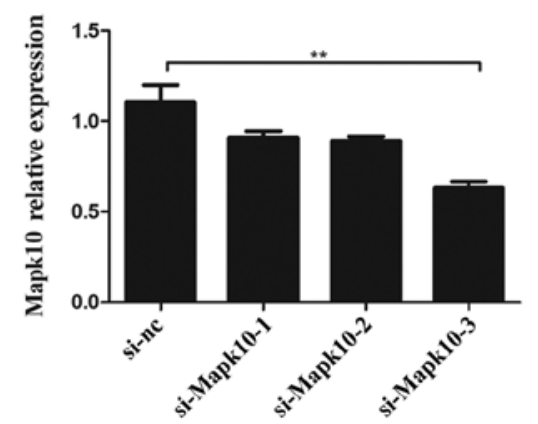

Figure 5. Mapk10 expression in NPC samples and cell lines. (A) RT-PCR analysis of Mapk10 was performed in the 5-8 F, 6-10B and HK-1 cell lines and in NP69 cells ( $\left.{ }^{*} \mathrm{p}<0.05\right)$. Compared to that in the control, the expression of Mapk10 was clearly downregulated in the 5-8 F cells. (B) Real-time RT-PCR analysis of Mapk10 was performed in 68 NPC patient samples and 21 control samples $(* \mathrm{*} p<0.01)$. Compared to that in the control samples, the expression of Mapk10 was clearly downregulated in the NPC samples. (C) Kaplan-Meier curves are shown for patients according to tumor expression of Mapk10. NPC death was significantly associated with the expression of Mapk10. Lower overall patient survival $(\mathrm{n}=48)$ was associated with low Mapk10 expression ( $\mathrm{p}=0.0418)$. (D) The correlation between the expression of miR-27a-3p and Mapk10 in 40 NPC patients. (E) The real-time PCR results verify the effectiveness of si-Mapk10. Si-Mapk10-3 was the most effective. NPC, nasopharyngeal carcinoma.

Mapk10 is a target of miR-27a-3p. To elucidate the molecular events underlying the increased miR-27a-3p expression in NPC cells, online algorithms for miRNA target prediction (PicTar, RNAhybrid and TargetScan) were used to search for putative miR-27a-3p target genes. Thousands of target genes were predicted for this miRNA. We compared the gene expression profiles of the NPC biopsies and the miRNA expression profiles performed as aforementioned. The expression data (GSE12452) from biopsies of NPC and non-malignant controls were downloaded from the National Center for Biotechnology Information-GEO. Putative target genes of miR-27a-3p that were differentially expressed in the NPC biopsies were identified. Then, the selected genes were analyzed in the context of Gene Ontology (GO) biological processes and the Kyoto Encyclopedia of Genes and Genomes (KEGG) biological pathways using the molecular annotations.

We found that the Mapk10 gene was a target of miR-27a-3p and was downregulated in the NPC samples. The DAVID results revealed that Mapk10 regulation involved the MAPK signaling pathway and pathways in cancer. We attempted to further ascertain these results. A schematic diagram of miR-27a-3p binding sites in the Mapk10 3'UTR is shown in Fig. 4A. To verify these binding sites, we constructed a luciferase reporter vector using the Mapk10 3'UTR, including both the wild-type sequence (WT Mapk10 3'UTR) and one in which the miR-27a-3p binding sites were deleted (MUT Mapk10 3'UTR) (Fig. 4A), and performed gene transfection and luciferase assays. We observed a significant decrease in the luciferase activity in the 5-8 $\mathrm{F}$ cells that overexpressed miR-27a-3p. In contrast, the luciferase activity of the construct with the MUT 3'UTR remained unchanged in the
miR-27a-3p-overexpressing 5-8 F cells (Fig. 4B). These results indicate that miR-27a-3p did bind to the WT Mapk 103'UTR but did not bind to the MUT Mapk10 3'UTR. We also performed qRT-PCR and western blotting experiments. The results of these experiments confirmed that miR-27a-3p overexpression inhibited the expression of endogenous Mapk10 mRNA and protein in the 5-8 F NPC cell line, whereas knockdown of miR-27a-3p expression upregulated endogenous Mapk10 expression (Fig. 4C and D).

Biological effect of miR-27a-3p is reversed by Mapk10. To explore the expression of Mapk 10 in NPC samples and cell lines, we detected the expression of Mapk10 in 68 NPC samples and 21 healthy nasopharyngeal epithelial tissue samples by quantitative real-time PCR. This analysis confirmed that Mapk10 was downregulated in the NPC samples compared to the healthy control samples (Fig. 5B). The expression of Mapk10 in the 5-8 F NPC cells was also downregulated compared to that in the NP69 cells (Fig. 5A). However, Mapk10 expression in the 6-10B and HK-1 cell lines did not differ from that in the NP69 cells. Kaplan-Meier plots were constructed based on the real-time PCR data $(n=48)$. Increased survival was associated with high Mapk10 expression (Fig. 5C, hazard ratio, 2.216; 95\% CI, 1.030-4.769; $\mathrm{p}=0.0418)$, demonstrating that death from NPC was associated with low levels of Mapk10 $(\mathrm{p}<0.05)$.

To validate that the carcinogenesis-promoting effect of miR-27a-3p depended on Mapk10, we examined the cell proliferation-promoting effect of miR-27a-3p on NPC cells with and without Mapk10 expression. The MTT assay results revealed that cell growth was obviously promoted by miR-27a-3p compared with the control. However, the cell 


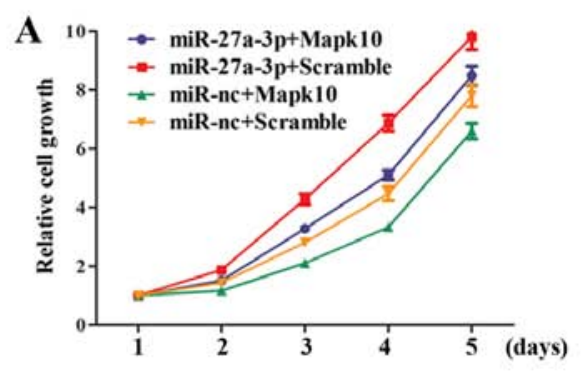

B

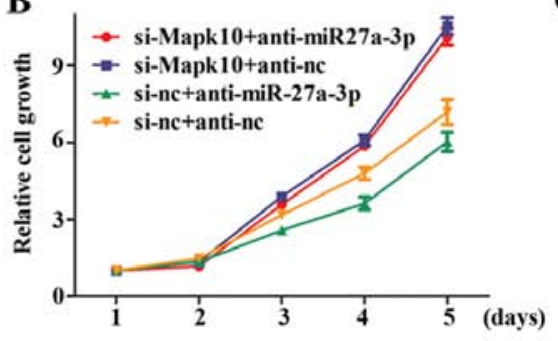

C

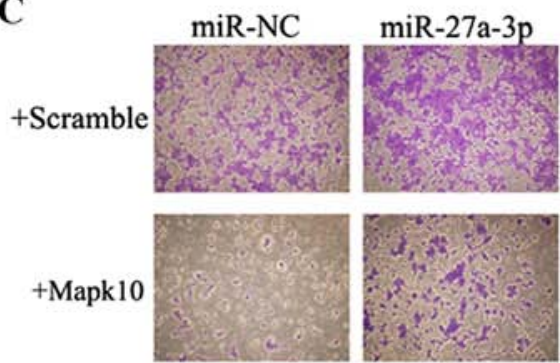

$\mathbf{E}$

D

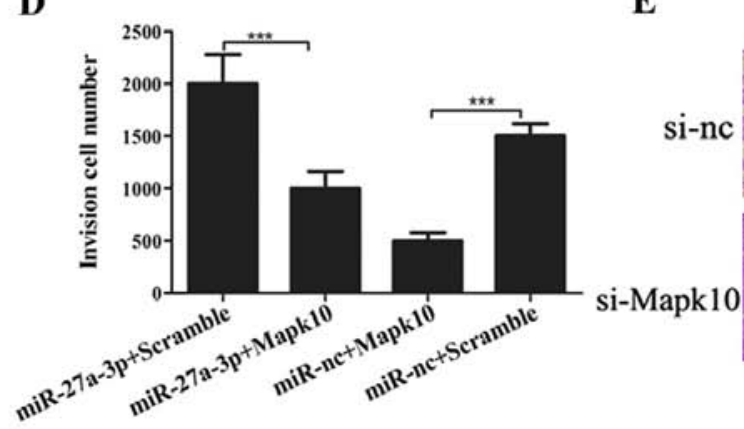

anti-miR nc anti-miR-27a-3p

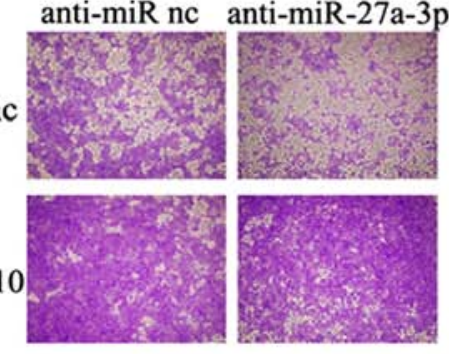

F

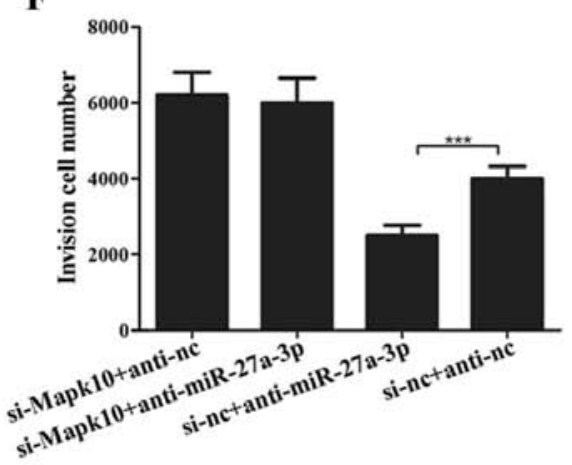

Figure 6. Function of miR-27a-3p in the promotion of 5-8 F cell proliferation and invasion partially depends on Mapk10. (A) The MTT cell viability assay revealed that miR-27a promoted cell viability through Mapk10. An MTT assay was performed after cotransfection of the miR-27a-3p mimics and the Mapk10 construct or control plasmid into 5-8 F cells. Mapk10 transfection decreased the cell proliferation induced by miR-27a-3p. The data represent the mean values of 3 experiments, each performed in triplicate. The data are shown as the mean \pm SD. (B) An MTT assay was performed after cotransfection of the miR-27a-3p and the Mapk10 siRNA. The growth-suppressing effect of the miR-27a-3p inhibitor was clearly attenuated when endogenous Mapk10 was knocked down. The data represent the mean \pm SD of 3 experiments, each performed in triplicate. (C) Transwell migration assays revealed that miR-27a-3p increased cell invasive ability through Mapk10. Representative images show the stained migrated cells. Mapk10 transfection clearly decreased the cell invasion induced by miR27a-3p. (D) The cells in five randomly selected fields in C were counted, and statistical analyses were performed using SPSS 22.0. The data are shown as the mean $\pm \mathrm{SD}\left({ }^{* * * *} \mathrm{p}<0.001\right.$; two-sided Student's t-test). (E) Transwell migration assays revealed that transfection of the miR-27a-3p inhibitor clearly decreased the number of migrated cells, but knockdown of endogenous Mapk10 partially reversed the inhibitory effect of miR-27a-3p. (F) The cells in five randomly selected fields in E were counted, and statistical analyses were performed using SPSS 22.0. The data are shown as the mean \pm SD (*** $p<0.001$; two-sided Student's t-test).

growth-promoting effect of miR-27a-3p was reversed by exogenous Mapk10 compared to the scrambled control (Fig. 6A). In contrast, treating the Mapk10-knockdown cells with antimiR-27a-3p did not have the antitumor growth effect observed in the intact 5-8 F cells (Fig. 6B). The Transwell with Matrigel (BD Biosciences) assay produced results similar to those of the MTT assay (Fig. 6C-F). These data indicated that miR-27a-3p promotes the growth and invasion of NPC cells, at least partially, by targeting Mapk10.

\section{Discussion}

miR-27a-3p is a member of the miR-23a 27-24a cluster. The role of the cluster, either oncogenic or suppressive, determines its function in disease. However, the expression levels of the 3 family members within the cluster under pathological conditions differ. In some diseases, miR-27a is significantly upregulated; for example, it is upregulated in laryngeal tumor tissues compared to adjacent non-tumor tissues. In addition, it acts as an oncogene in laryngeal squamous cell carcinoma through downregulation of PLK2 (27). Liu et al further revealed that miR-27b upregulation by the human papillomavirus $16 \mathrm{E} 7$ promotes proliferation and suppresses apoptosis by targeting polo-like kinase 2 in cervical cancer (28). miR-27a-3p was also found to promote esophageal cancer cell proliferation via F-box and WD repeat domain-containing 7 (FBXW7) suppression (29).
However, conflicting results regarding the function of miR-27a have also been reported. For example, EGFR was found to be a novel target of miR-27a in renal cell carcinoma (30), and miR-27a-3p was reported to inhibit melanogenesis by suppressing Wnt3a at the post-transcriptional level (31). The expression of miR-27a-3p in NPC was confirmed by Illumina Hiseq2000 deep sequencing of laser-microdissected biopsies from 12 NPC and 8 chronic nasopharyngitis patients (23). However, there were no data concerning the association of miR-27a-3p expression with NPC TNM or prognosis. In the present study, we also found that the expression of miR-27a-3p was upregulated in NPC using miRNA array chips (Fig. 1). We also confirmed the miR-27a-3p expression results by quantitative real-time PCR in 68 NPC samples and 21 healthy nasopharyngeal epithelial tissue samples. We found that the expression level of miR-27a-3p in the NPC samples gradually increased with clinical stage (Table I). The Kaplan-Meier plots also showed that low expression of miR-27a-3p was associated with high survival (Fig. 2C), demonstrating that death from NPC was associated with high levels of miR-27a-3p $(p<0.05)$. We also examined the association of the characteristics of 68 NPC samples, including age, gender, lymph node metastasis, clinical stage and EBV (VCA-IgA), with the expression of miR-27a-3p (Table I).

Furthermore, the MTT assay revealed that miR-27a-3p overexpression increased cell viability. In contrast, the 
miR-27a-3p inhibitor (anti-miR-27a-3p) obviously inhibited the growth compared to the control-treated cells (Fig. 3A). The wound healing (Fig. 3B) assay and Transwell invasion assay (Fig. 3C and D) also revealed that miR-27a-3p promoted NPC cell proliferation, migration and invasion in vitro more than the controls. In contrast, the miR-27a-3p inhibitor obviously inhibited the growth and invasion of the NPC cells. These results indicate that miR-27a-3p may play an important role in the progression of NPC.

Bioinformatics algorithms have played a key role in the discovery of miRNA target genes. MiRNAome, TargetScan, PicTar and miRanda are commonly used to predict miRNA target genes. However, the targets predicted by these algorithms are not always consistent. It is necessary to further confirm that the predicted target genes are related to the regulatory function of the miRNA. In the present study, the thousands of predicted target genes were refined by screening specifically for nasopharyngeal genes. Another data reduction strategy is to compare the miRNA expression and cDNA expression data and select the miRNAs whose expression is consistent with the cDNA expression. The cDNA expression data (GSE12452) were downloaded from the publicly accessible GEO Data. Using these data reduction strategies, we identified 224 mRNAs that were downregulated in NPC as potential target genes of miR-27a-3p. The subsequent luciferase reporter experiment confirmed that Mapk10 was a target of miR-27a-3p. Exogenous miR-27a-3p inhibited the expression of endogenous Mapk10 mRNA and protein, whereas knockdown of miR-27a-3p expression upregulated endogenous Mapk10 expression (Fig. 4C and D).

Mapk10 is a member of the MAP kinase family. MAP kinases act as integration points for multiple biochemical signals and are involved in a wide variety of cellular processes, such as proliferation, differentiation, transcription regulation and development $(32,33)$. Targeted deletion of this gene in mice suggests that it may have a role in stress-induced neuronal apoptosis (34). However, spliced transcript variants encoding different isoforms have also been described for this gene. Yoo et al showed that Mapk10 is a novel epigenetic marker in chromophobe kidney cancer. Ying et al (35) reported that the expression of Mapk10 and PTPN13 was frequently downregulated or silenced in non-Hodgkin's lymphoma (94\%, 15 of 16), Hodgkin's lymphoma (50\%, 3 of 6$)$, breast (30\%, 3 of 10), gastric $(60 \%, 6$ of 10) and hepatocellular (67\%, 8 of 12) carcinoma cell lines; however, Mapk10 expression in the CNE1, CNE2, and HK1 NPC cell lines did not differ from that in control cells. In the present study, we found low expression of Mapk10 in 48 NPC and 21 control samples. The Kaplan-Meier plots showed that increased survival was associated with low expression of Mapk10 (Fig. 5C, hazard ratio, 2.216; 95\% CI, 1.030-4.769; $\mathrm{p}=0.0418, \mathrm{n}=48$ ), demonstrating that death from NPC was associated with low levels of Mapk10 $(\mathrm{p}<0.05)$. These results were also consistent with the GEO data (GSE12452) for 25 NPC and 3 control samples.

In the present study, miR-27a-3p was found to be upregulated in NPC, and its expression level was associated with lymph node metastasis, clinical stage and EBV (VCA-IgA). miR-27a-3p also promoted 5-8 F growth and mobility, an effect that at least partially depended on Mapk10.

\section{Acknowledgements}

The present study was supported by funding from the National Natural Science Foundation, China (grant no. 81301024), the Hunan Natural Science Foundation (grant no. 14JJ3033), and the post-doctoral fund of China (nos. 2014M550427 and 2015T80892).

\section{References}

1. Lu J, Getz G, Miska EA, Alvarez-Saavedra E, Lamb J, Peck D, Sweet-Cordero A, Ebert BL, Mak RH, Ferrando AA, et al: MicroRNA expression profiles classify human cancers. Nature 435: 834-838, 2005.

2. Hanahan D and Weinberg RA: Hallmarks of cancer: The next generation. Cell 144: 646-674, 2011.

3. Yang LH, Dong Z and Gong ZH: Extracellular miRNA: A novel molecular biomarker for lung cancer. Yi Chuan 34: 651-658, 2012 (In Chinese)

4. Gao W, Lu X, Liu L, Xu J, Feng D and Shu Y: MiRNA-21: A biomarker predictive for platinum-based adjuvant chemotherapy response in patients with non-small cell lung cancer. Cancer Biol Ther 13: 330-340, 2012.

5. Ebert MS and Sharp PA: Roles for microRNAs in conferring robustness to biological processes. Cell 149: 515-524, 2012.

6. Bartel DP: MicroRNAs: Target recognition and regulatory functions. Cell 136: 215-233, 2009.

7. Bowen T, Jenkins RH and Fraser DJ: MicroRNAs, transforming growth factor beta-1, and tissue fibrosis. J Pathol 229: 274-285, 2013.

8. Zhou L, Liang X, Zhang L, Yang L, Nagao N, Wu H, Liu C, Lin S, Cai G and Liu J: MiR-27a-3p functions as an oncogene in gastric cancer by targeting BTG2. Oncotarget 7: 51943-51954, 2016.

9. Guo D, Li Q, Lv Q, Wei Q, Cao S and Gu J: MiR-27a targets sFRP1 in hFOB cells to regulate proliferation, apoptosis and differentiation. PLoS One 9: e91354, 2014.

10. Urbich C, Kaluza D, Frömel T, Knau A, Bennewitz K, Boon RA, Bonauer A, Doebele C, Boeckel JN, Hergenreider E, et al: MicroRNA-27a/b controls endothelial cell repulsion and angiogenesis by targeting semaphorin 6A. Blood 119: 1607-1616, 2012.

11. Deng M, Tang H, Zhou Y, Zhou M, Xiong W, Zheng Y, Ye Q, Zeng X, Liao Q, Guo X, et al: miR-216b suppresses tumor growth and invasion by targeting KRAS in nasopharyngeal carcinoma. $J$ Cell Sci 124: 2997-3005, 2011.

12. Luo Z, Dai Y, Zhang L, Jiang C, Li Z, Yang J, McCarthy JB, She $\mathrm{X}$, Zhang W, Ma J, et al: miR-18a promotes malignant progression by impairing microRNA biogenesis in nasopharyngeal carcinoma. Carcinogenesis 34: 415-425, 2013.

13. Alajez NM, Lenarduzzi M, Ito E, Hui AB, Shi W, Bruce J, Yue S, Huang SH, Xu W, Waldron J, et al: MiR-218 suppresses nasopharyngeal cancer progression through downregulation of survivin and the SLIT2-ROBO1 pathway. Cancer Res 71: 2381-2391, 2011.

14. Lu J, He ML, Wang L, Chen Y, Liu X, Dong Q, Chen YC, Peng Y, Yao KT, Kung HF, et al: MiR-26a inhibits cell growth and tumorigenesis of nasopharyngeal carcinoma through repression of EZH2. Cancer Res 71: 225-233, 2011.

15. Ji Y, He Y, Liu L and Chong X: MiRNA-26b regulates the expression of cyclooxygenase-2 in desferrioxamine-treated CNE cells. FEBS Lett 584: 961-967, 2010.

16. Li G, Wu Z, Peng Y, Liu X, Lu J, Wang L, Pan Q, He ML and Li XP: MicroRNA-10b induced by Epstein-Barr virus-encoded latent membrane protein-1 promotes the metastasis of human nasopharyngeal carcinoma cells. Cancer Lett 299: 29-36, 2010.

17. Wong TS, Man OY, Tsang CM, Tsao SW, Tsang RK, Chan JY, Ho WK, Wei WI and To VS: MicroRNA let-7 suppresses nasopharyngeal carcinoma cells proliferation through downregulating c-Myc expression. J Cancer Res Clin Oncol 137: 415-422, 2011.

18. Zhang L, Deng T, Li X, Liu H, Zhou H, Ma J, Wu M, Zhou M, Shen S, Li X, et al: microRNA-141 is involved in a nasopharyngeal carcinoma-related genes network. Carcinogenesis 31 : 559-566, 2010.

19. Xia H, Ng SS, Jiang S, Cheung WK, Sze J, Bian XW, Kung HF and Lin MC: miR-200a-mediated downregulation of ZEB2 and CTNNB1 differentially inhibits nasopharyngeal carcinoma cell growth, migration and invasion. Biochem Biophys Res Commun 391: 535-541, 2010. 
20. Gourzones C, Jimenez AS and Busson P: Profiling of Epstein-Bar virus-encoded microRNAs in nasopharyngeal carcinoma reveals potential biomarkers and oncomirs. Cancer 118: 4634, author reply 4634-4635, 2012.

21. Xie YJ, Long ZF and He XS: Involvement of EBV-encoded BART-miRNAs and dysregulated cellular miRNAs in nasopharyngeal carcinoma genesis. Asian Pac J Cancer Prev 14: 5637-5644, 2013.

22. Barth S, Meister G and Grässer FA: EBV-encoded miRNAs Biochim Biophys Acta 1809: 631-640, 2011.

23. Wang F, Lu J, Peng X, Wang J, Liu X, Chen X, Jiang Y, Li X and Zhang B: Integrated analysis of microRNA regulatory network in nasopharyngeal carcinoma with deep sequencing. J Exp Clin Cancer Res 35: 17, 2016.

24. Luo Z, Zhang L, Li Z, Li X, Li G, Yu H, Jiang C, Dai Y, Guo X, $\mathrm{Xiang} \mathrm{J}$, et al: An in silico analysis of dynamic changes in microRNA expression profiles in stepwise development of nasopharyngeal carcinoma. BMC Med Genomics 5: 3, 2012.

25. Cho JH, Gelinas R, Wang K, Etheridge A, Piper MG, Batte K, Dakhallah D, Price J, Bornman D, Zhang S, et al: Systems biology of interstitial lung diseases: Integration of mRNA and microRNA expression changes. BMC Med Genomics 4: 8, 2011.

26. Zungu IL, Mbene AB, Hawkins Evans DH, Houreld NN and Abrahamse H: Phototherapy promotes cell migration in the presence of hydroxyurea. Lasers Med Sci 24: 144-150, 2009.

27. Tian Y, Fu S, Qiu GB, Xu ZM, Liu N, Zhang XW, Chen S, Wang Y, Sun KL and Fu WN: MicroRNA-27a promotes proliferation and suppresses apoptosis by targeting $P L K 2$ in laryngeal carcinoma. BMC Cancer 14: 678, 2014.

28. Liu F, Zhang S, Zhao Z, Mao X, Huang J, Wu Z, Zheng L and Wang Q: MicroRNA-27b up-regulated by human papillomavirus 16 E7 promotes proliferation and suppresses apoptosis by targeting polo-like kinase 2 in cervical cancer. Oncotarget 7 : 19666-19679, 2016.
29. Wu XZ, Wang KP, Song HJ, Xia JH, Jiang Y and Wang YL: MiR-27a-3p promotes esophageal cancer cell proliferation via F-box and WD repeat domain-containing 7 (FBXW7) suppression. Int J Clin Exp Med 8: 15556-15562, 2015.

30. Li Y, Li J, Sun X, Chen J, Sun X, Zheng J and Chen R: MicroRNA-27a functions as a tumor suppressor in renal cell carcinoma by targeting epidermal growth factor receptor. Oncol Lett 11: 4217-4223, 2016.

31. Zhao Y, Wang P, Meng J, Ji Y, Xu D, Chen T, Fan R, Yu X, Yao J and Dong C: MicroRNA-27a-3p inhibits melanogenesis in mouse skin melanocytes by targeting Wnt3a. Int J Mol Sci 16: 10921-10933, 2015.

32. Kunde SA, Rademacher N, Tzschach A, Wiedersberg E, Ullmann R, Kalscheuer VM and Shoichet SA: Characterisation of de novo MAPK10/JNK3 truncation mutations associated with cognitive disorders in two unrelated patients. Hum Genet 132: 461-471, 2013.

33. Yoo KH, Park YK, Kim HS, Jung WW and Chang SG: Identification of MAPK10 as a novel epigenetic marker for chromophobe kidney cancer. Pathol Int 61: 52-54, 2011.

34. Gourmaud S, Paquet C, Dumurgier J, Pace C, Bouras C, Gray F, Laplanche JL, Meurs EF, Mouton-Liger F and Hugon J: Increased levels of cerebrospinal fluid JNK3 associated with amyloid pathology: Links to cognitive decline. J Psychiatry Neurosci 40: 151-161, 2015.

35. Ying J, Li H, Cui Y, Wong AH, Langford C and Tao Q: Epigenetic disruption of two proapoptotic genes MAPK10/JNK3 and PTPN13/FAP-1 in multiple lymphomas and carcinomas through hypermethylation of a common bidirectional promoter. Leukemia 20: 1173-1175, 2006 